\title{
Alignment of the ATLAS inner detector for the LHC Run II
}

\author{
Butti Pierfrancesco ${ }^{* \dagger}$ \\ NIKHEF \\ E-mail: pierfrancesco.buttiecern.ch
}

\begin{abstract}
ATLAS is a multipurpose experiment at the LHC proton-proton collider. Its physics goals require high resolution, unbiased measurement of all charged particle kinematic parameters. These critically depend on the layout and performance of the tracking system, quality of its offline alignment. ATLAS is equipped with a tracking system built using different technologies, silicon planar sensors (pixel and micro-strip) and gaseous drift-tubes, all embedded in a $2 \mathrm{~T}$ magnetic field provided by a solenoid. For the LHC Run II, the system has been upgraded with the installation of a new pixel layer, the Insertable B-layer (IBL). Offline track alignment of the ATLAS tracking system has to deal with about 700,000 degrees of freedom (DoF) defining its geometrical parameters. The task requires using very large data sets and represents a considerable numerical challenge in terms of both CPU time and precision. The adopted strategy uses a hierarchical approach to alignment, combining local and global least squares techniques. An outline of the track based alignment approach and its implementation within the ATLAS software will be presented. Special attention will be paid to integration to the alignment framework of the IBL, which plays the key role in precise reconstruction of the collider luminous region, interaction vertices and identification of long-lived heavy flavor states. Techniques allowing to pinpoint and eliminate tracking systematics due to alignment as well as strategies to deal with time-dependent variations will be briefly covered. The first results from cosmic ray commissioning runs will be discussed. During the commissioning data taking a mechanical distortion of the IBL staves has been observed and a preliminary study of the mechanical stability will be presented.
\end{abstract}

24th International Workshop on Vertex Detector -VERTEX2015-

\section{1-5 June 2015}

Santa Fe, New Mexico, USA

\footnotetext{
* Speaker.

${ }^{\dagger}$ on behalf of the ATLAS collaboration
} 


\section{Inner Detector Structure}

The ATLAS [1] Inner Detector (ID) [2] consists of three subdetectors, the Pixel detector including the new IBL, the Semiconductor Tracker (SCT) and the Transition Radiation Tracker (TRT), all embedded in a $2 \mathrm{~T}$ axial magnetic field and designed to reconstruct charged particles within a pseudorapidity range of $|\eta|<2.5$. In Fig. 1 is shown a schematic representation of the ATLAS Inner Detector. The Pixel sub-detector consists of 1744 silicon pixel modules arranged in three barrel layers and two end caps with three disks each. The expected detector measurement resolution is $10 \mu \mathrm{m}$ in $r-\phi^{1}$ (local $x$ ) and $115 \mu \mathrm{m}$ in $z$ (local $y$ ). During the long shutdown one (LS1) the IBL $[3,4]$ has been added as an additional layer to the Pixel detector, reducing the distance from the interaction point to the first tracking layer. It consists of 280 silicon pixel modules arranged on 14 azimuthal carbon fiber staves surrounding the beam pipe at a mean radius of 33.2 mm. Each stave is instrumented with 12 two-chip planar modules, covering the region of $|\eta|<$ 2.7, and 8 single chip modules with 3D sensors, four at each end of the stave $(2.7<|\eta|<3)$. The sensitive element size is $50 \mu \mathrm{m}$ in $r-\phi$ and $250 \mu \mathrm{m}$ in $z$. In order to simplify the notation throughout the remainder of the note, the term Pixel will be used to refer only to the 50x400 $\mu \mathrm{m}$ pixels and IBL to the new layer. The SCT consists of 4088 silicon strip modules, arranged in four barrel layers and two end caps with nine wheels each. The intrinsic resolution is $\sim 17 \mu \mathrm{m}$ in $r-\phi$. The TRT is the outermost of the ID subdetectors, consists of a barrel and two end caps and is made of 350848 gas-filled straw tubes with a single hit resolution of $\sim 130 \mu \mathrm{m}$ along $r-\phi$.

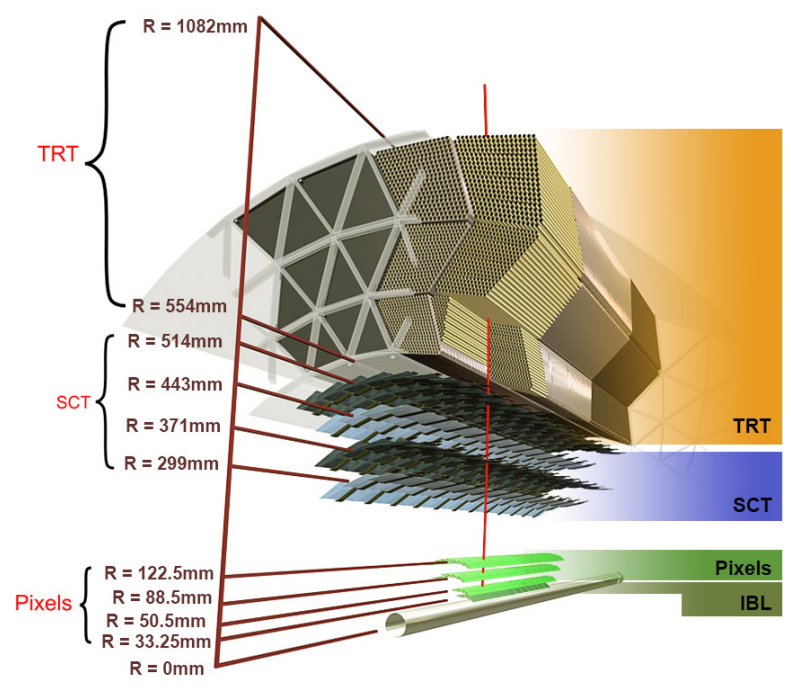

Figure 1: A 3D visualisation of the structure of the barrel of the Inner Detector. In the picture are shown the beam pipe, the IBL, the Pixel layers, the four cylindrical layers of the SCT and the 72 straw layers of the TRT.

\footnotetext{
${ }^{1}$ ATLAS uses a right-handed coordinate system with its origin at the nominal interaction point in the centre of the detector and the $z$-axis along the beam pipe. The $x$-axis points from the interaction point to the centre of the LHC ring, and the $y$-axis points upwards. Cylindrical coordinates $(r, \phi)$ are used in the transverse plane, $\phi$ being the azimuthal angle around the beam pipe.
} 


\section{Inner Detector Alignment Procedure}

The alignment of the ATLAS Inner Detector is performed using a track-based technique, which minimises the track-to-hit residuals, and obtains the corrections to the module positions of the detector. Each module or grouped collection of modules, e.g. a sub-detector, can be treated as an alignable structure. Each structure has six degrees of freedom (DOF), three rotations and three translations, that define its position and orientation in space. The DOF are referred to as alignment parameters $(\boldsymbol{a})$. The alignment algorithms construct a $\chi^{2}$ defined as:

$$
\chi^{2}=\sum_{t r k}\left[\boldsymbol{r}^{T}(\boldsymbol{\tau}, \boldsymbol{a}) V^{-1} \boldsymbol{r}(\boldsymbol{\tau},(\boldsymbol{a}))\right]
$$

where $V$ is the covariance matrix of the detector measurements, $\boldsymbol{r}$ is the track-to-hit residuals depending on the alignment parameters and the track parameters $\tau$. Then a minimisation is performed:

$$
\frac{d \chi^{2}}{d \mathbf{a}}=0 \rightarrow \sum_{t r k}\left[\mathbf{r}^{T} V^{-1}\left(\frac{\partial \mathbf{r}}{\partial \boldsymbol{\tau}} \frac{d \boldsymbol{\tau}}{d \mathbf{a}}+\frac{\partial \mathbf{r}}{\partial \mathbf{a}}\right)\right]=0
$$

In order to cope with a large number of degrees of freedom, the ID alignment is performed in three hierarchical Levels: at Level 1 seven physical structures are aligned, the Pixel as a whole, the SCT barrel and the two end caps, the TRT barrel and, finally, the two TRT end caps. The Level 2 treats the silicon barrel layers and end cap disks and TRT barrel modules and end caps wheels as separate objects. The Level 3 aligns all the silicon modules and the individual wires of the TRT. The alignment framework has been extensively updated in order to cope with the integration of the new IBL sub-detector in the ID tracking system. A new alignment Level has been defined, Level 11, that treated the IBL as a separate structure from the Pixel detector [5]. This alignment procedure is run during ATLAS data acquisition to monitor the global stability of the ATLAS Inner Detector [6]. Any generic track-based alignment is confronted with some deformations to which fitted tracks have very low or no sensitivity. These, so called weak modes of alignment are collective detector deformations that transform a helical trajectory of a track to another helical trajectory, hence do not affect the $\chi^{2}$ of the track fit. A number of updates have been applied to the ID alignment to correct for weak mode deformations during the Run I alignment campaign [7].

\section{Inner Detector Alignment Commissioning with Cosmic Data}

Data recorded by ATLAS during 2015 campaign of cosmic rays have been used to perform a first alignment of the detector after the LS1. Around $3 \times 10^{5}$ tracks were reconstructed in the data taken in February 2015, which have been used for the first alignment of the ATLAS Inner Detector. The details about the track selection and the alignment procedure can be found in [8]. Using the data recorded in March 2015, the previous results have been improved. Firstly, using data collected with turned off magnetic field, an alignment up to stave level has been performed. The advantage of using magnetic field off data relies on exploiting straight tracks for deriving alignment corrections that are more robust against the introduction of weak modes. In this configuration no information on the track momentum is available. Therefore in order to reduce the uncertainty on 
the determination of the alignment parameters due to material effects when using low momenta tracks, the Pixel and IBL module level alignment has been performed only using data collected with turned on magnetic field. A cut on the $p_{T}>2 \mathrm{GeV}$ has been applied in order to remove tracks with hit resolution uncertainty dominated by multiple coulomb scattering. The track-to-hit residual distributions before and after the Inner Detector alignment for the IBL and the outer three layers of the pixel barrel are shown in Fig. 2 and Fig. 3. After the module level March alignment of the IBL and Pixel subdetectors, the hit resolution of the IBL has been improved in local $x$ (local $y$ ) from 197 (153) $\mu \mathrm{m}$ to 22 (82) $\mu \mathrm{m}$ respectively and a misalignment of 40 (28) $\mu \mathrm{m}$ has been fully corrected.
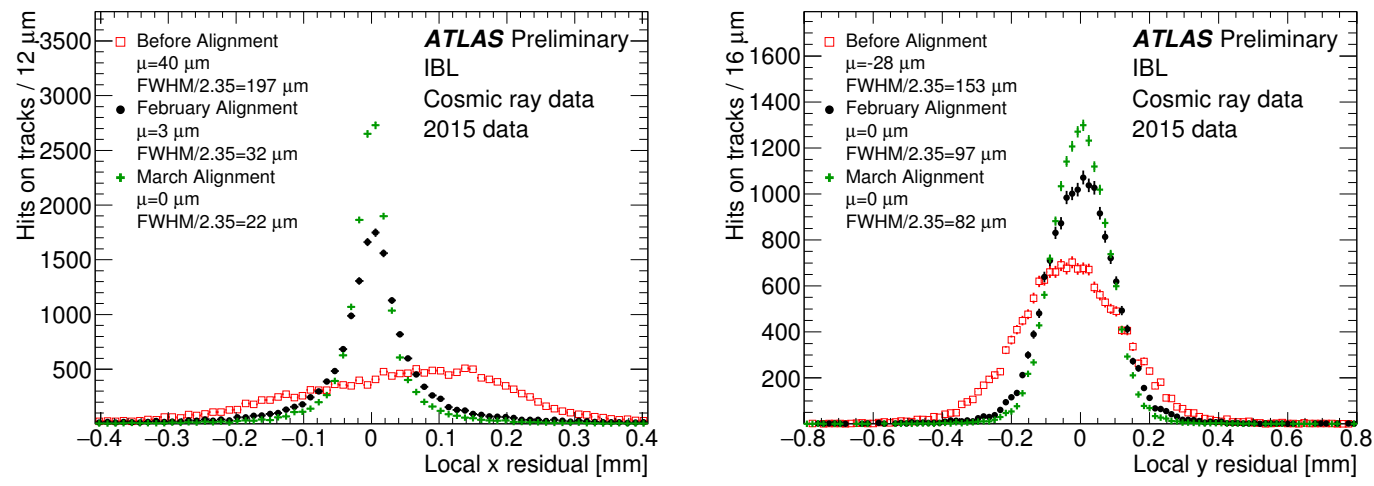

Figure 2: The IBL local $x$ (left) and local $y$ (right) residual distribution for the cosmic-ray data sample reconstructed before (red), after February (black) and March (green) alignment [8]. The distributions are integrated over all hits-on-tracks in IBL modules. The parameter $\mu$ represents the mean of the distributions.
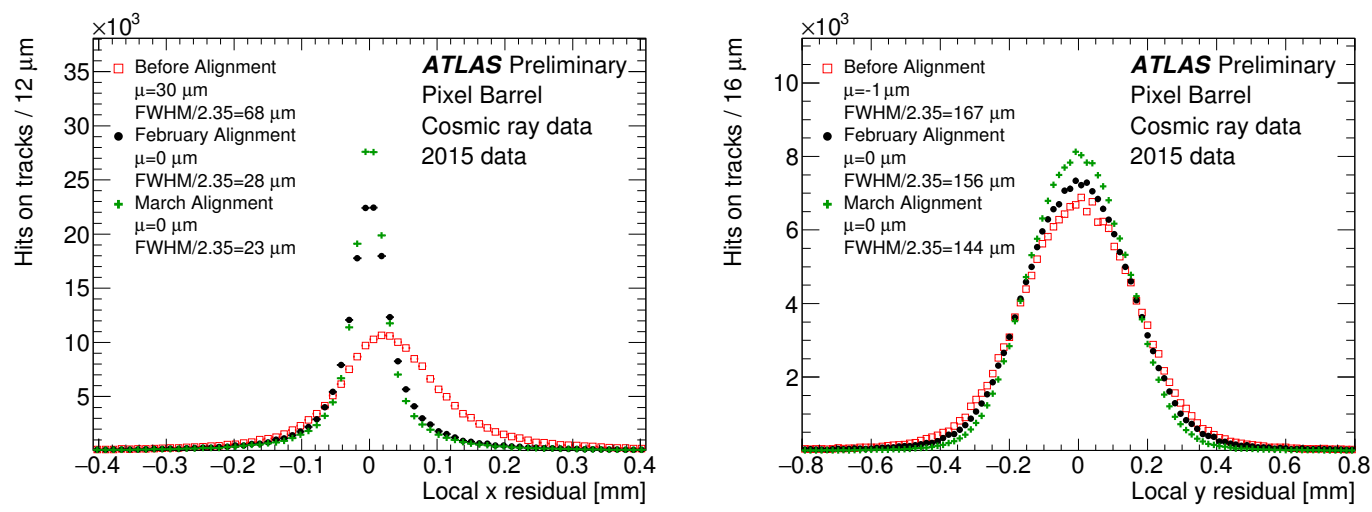

Figure 3: The Pixel local $x$ (left) and local $y$ (right) residual distribution for the cosmic-ray data sample reconstructed before (red), after February (black) and after March (green) alignment [8]. The distributions are integrated over all hits-on-tracks in the barrel modules of pixel layer one, two and three. The parameter $\mu$ represents the mean of the distributions.

The means of the local $x$ (left) and local $y$ (right) residual distributions as a function of the module location along the stave ( $\eta$-index), integrated over all 14 IBL staves are shown in Fig. 4. The distribution of the local $x$ residual mean indicates an in-plane deformation (stave bowing) in the negative local $x$ direction, with respect to the nominal geometry. The causes of this deformation will be described in more detail in section 4. The distribution in local $y$ indicates a systematic 
displacement of the modules along the beam axis. As all the IBL modules have been glued on the staves from the stave-center outwards, such stave elongation with respect to the nominal geometry has been ascribed to a wider intermediary gap between the neighbouring modules. The module level alignment corrects both observed stave deformations.
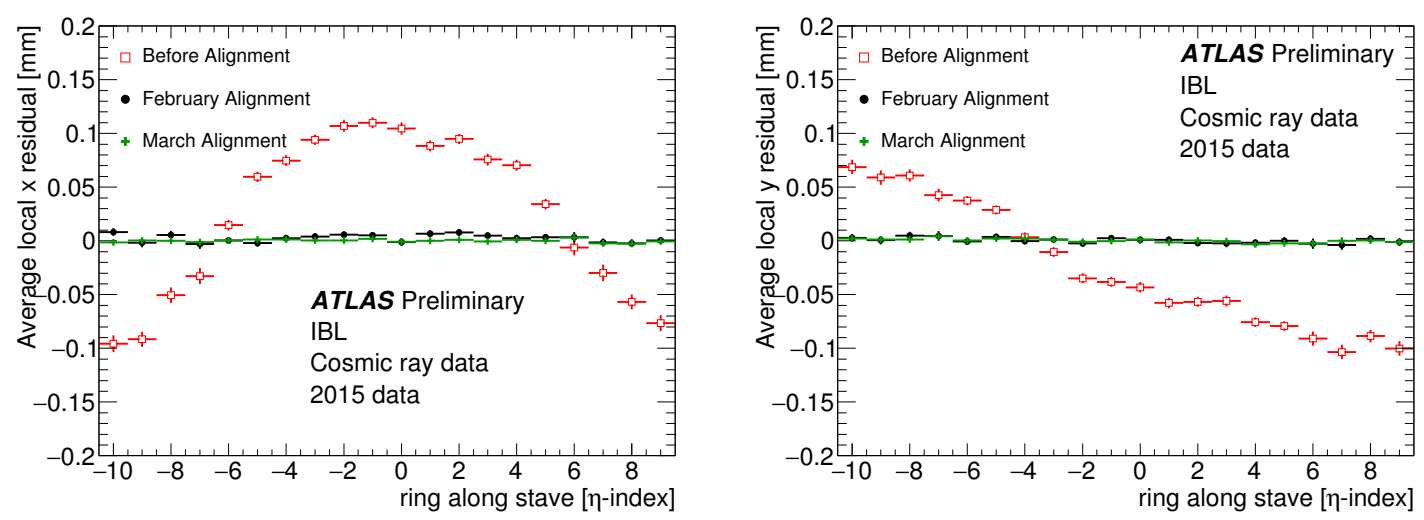

Figure 4: The IBL mean of the local $x$ (left) and local $y$ (right) residual distributions as a function of the $\eta$ position of the module [8]. The distributions before (red), after February (black) and after March (green) alignment are shown.

The half track method [8] has been used to split the cosmic tracks that go through the whole Inner Detector, using respectively the hits in the top and bottom halves. The perigee parameters $\tau_{u p}$ and $\boldsymbol{\tau}_{\text {down }}$ of each half track pair are compared to each other and their difference, $\Delta\left(\tau_{u p}-\tau_{\text {down }}\right)$, is compared before and after the alignment. The resolution of a track parameter is obtained by the width of the distribution of $\Delta\left(\boldsymbol{\tau}_{u p}-\boldsymbol{\tau}_{\text {down }}\right)$ divided by $\sqrt{2}$. The distributions of the difference between the split tracks longitudinal and transverse impact parameters, the azimuthal angle at the track perigee and the track charge over transverse momentum are reported in Fig. 5.

\section{Temperature Distortion of the IBL}

The distortion of the IBL observed in the data (see previous section) turned out to depend on a mismatch between the coefficient of thermal expansion (CTE) of the components of the IBL staves. Detailed investigations of the characteristic of this distortion are reported in [9].

\subsection{Stave Bowing Simulation and Measurement}

A three-dimensional finite element analysis (FEA) has been performed to investigate the observed distortion of the IBL staves. The FEA considers the detailed structure of the staves and implements all the mechanical constraints acting on the staves' shape and the boundary condition at the fixation points. The FEA simulation shows that the stave bows to the negative $\phi$ direction in the ATLAS global coordinate system when it is cooled down, as shown in Fig. 6 (left). The stave bowing is due to the difference of the CTE of the bare stave, that is almost zero ppm per Kelvin, and the one of the polyamide flex bus line, that is several tens of ppm per Kelvin. The FEA simulation calculated that the magnitude of the bowing is expected to be approximately parabolic and the distortion size is proportional to the difference between the operating temperature and the 

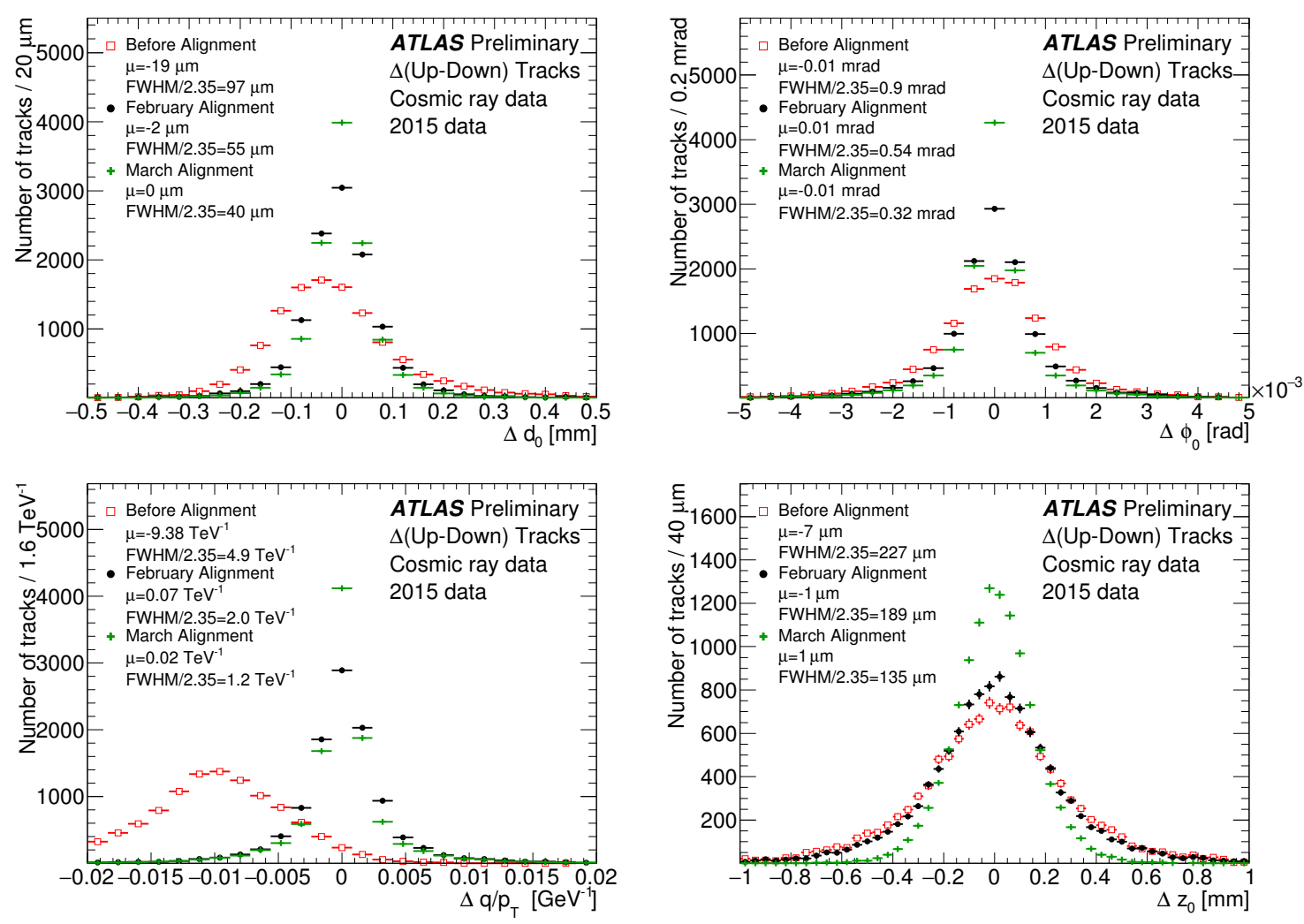

Figure 5: Distribution of the difference of the reconstructed track transverse impact parameter $\Delta d_{0}$ (top left), of the azimuthal angle at the track perigee $\Delta \phi_{0}$ (top right), of the difference of the reconstructed track charge over transverse momentum $\Delta q / p_{T}$ (bottom left) and of the longitudinal impact parameter $\Delta z_{0}$ (bottom right) using tracks reconstructed in the top part of the inner detector with respect to track reconstructed in the bottom part. The distributions before (red), after February (black) and March (green) alignment are shown [8].

nominal. The measurement of the correlation of the size of IBL distortion to the operating temperature has been performed using cosmic ray data collected in March 2015. The IBL distortion has been quantified determining the mean of the track-to-hit residual distributions as a function of the module position in global $z$, integrated over the 14 staves. The cosmic ray data has been collected at different IBL operating points and the resulting distributions are shown in Fig. 6 (right). The track-to-hit residual distributions have been fit using a parabolic formula

$$
\Delta_{x_{L}}(z)=B-\frac{M}{z_{0}^{2}}\left(z^{2}-z_{0}^{2}\right)
$$

where $\Delta_{x_{L}}(z)$ is the in-plane module displacement as a function of the global $z$ position, $B$ is the fit baseline, $M$ is a free parameter that represent the bowing magnitude and $z_{0}=366.5 \mathrm{~mm}$ is the fixing point of the stave at both ends. The magnitude of the distortion as function of the operating temperature is shown in Fig. 7 (left). The slope of the linear fit is

$$
\frac{d M}{d T}=-10.6 \pm 0.7[\mu m / K]
$$


The observation supports the hypothesis that the distortion is driven by the mismatch of CTEs of the materials composing the IBL staves.
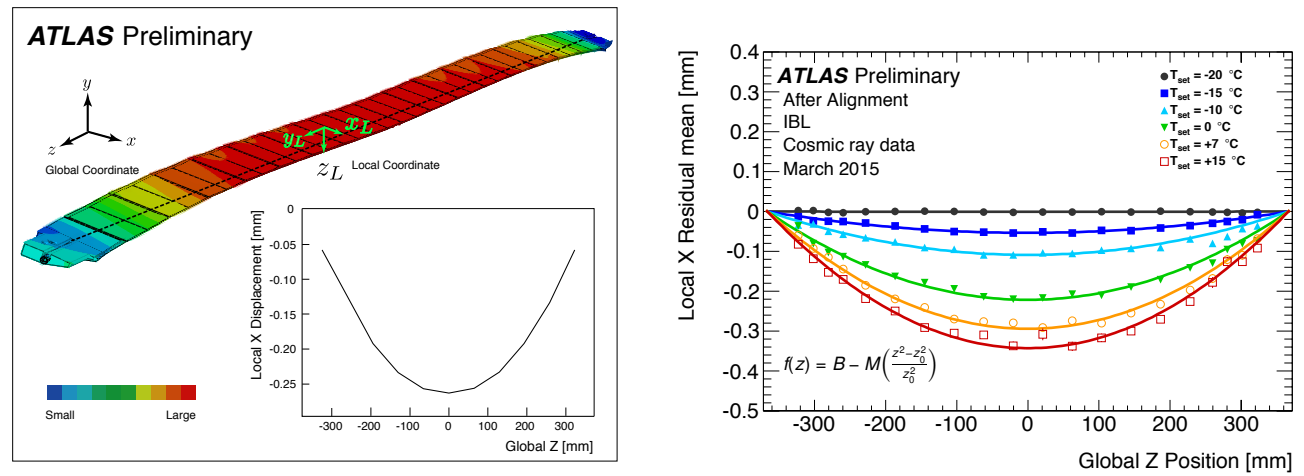

Figure 6: (left) Visualisation of the distorted stave with magnified distortion size. The size of the distortion is magnified for visualisation. The colour represents the magnitude of the displacement. The right bottom graph shows the relative displacement size in local $x$ direction $\left(x_{L}\right)$ as a function of the global $z$-position at the face plate surface of the stave. (right) The track-to-hit residual mean in the local $x$ direction. The residual mean is averaged over all hits of modules at the same global- $z$ position. Each data set is fitted to a parabola which is constrained to match to the baseline $B=0$ at $z= \pm z_{0}= \pm 366.5 \mathrm{~mm}$ [9].
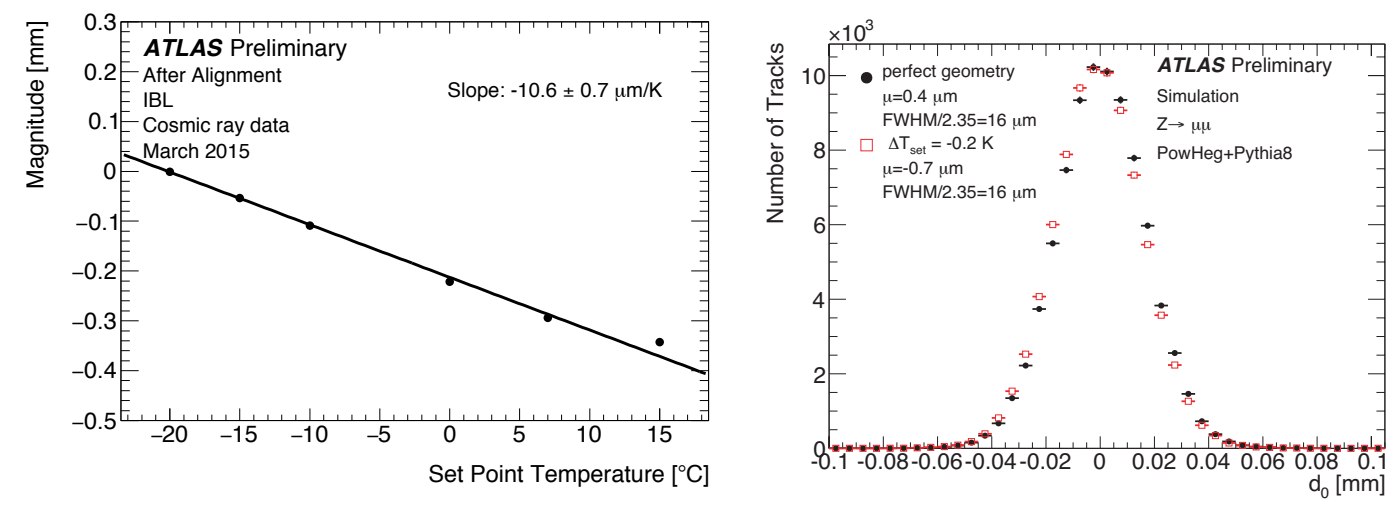

Figure 7: (left) The magnitude of the distortion as a function of the temperature set point. Each data point is a best fit of a parabola to the local $x$ residual mean as function of the global- $z$ of the module position. The alignment corrections derived at $-20^{\circ} \mathrm{C}$ are applied to the local positions in the module frames. (right) Distribution of the transverse impact parameter $d_{0}$ of tracks with respect to the beamspot from $Z \rightarrow \mu \mu$ events simulated in $\sqrt{s}=13 \mathrm{TeV} p p$ collision. Solid circle shows the nominal geometry and the open square shows the distorted geometry corresponding to a temperature variation of the IBL at $-0.2 \mathrm{~K}(\sim 2 \mu \mathrm{m}$ of displacement at the center of the stave) [9].

\subsection{Impact over Track Parameters}

A set of alignment constants has been produced to mimic the IBL stave distortion in the local $x$ axis, in order to determine the effect on the track reconstruction. Due to the positioning of the IBL inside the Inner Detector, it is expected that the transverse impact parameter $\left(d_{0}\right)$ of a track is 
the most sensitive track parameter to the stave distortion. The comparison of the $d_{0}$ distributions of muon tracks using the Monte Carlo simulation of $Z \rightarrow \mu^{+} \mu^{-}$events in $\sqrt{s}=13 \mathrm{TeV}$ proton-proton collisions reconstructed with the nominal geometry and the distorted geometry is shown in Fig. 7 (right). The track selection is described in detail in [9]. A bias of $\sim 1 \mu \mathrm{m}$ has been observed by comparing the distributions' mean values in the case of a distortion magnitude corresponding to $\delta T=-0.2 \mathrm{~K}^{2}$, which is very small compared to the $d_{0}$ distribution resolution.

\section{Conclusions}

The ID alignment framework has been upgraded to cope with the Run II requirements. A first alignment of the ID after the LS1 has been performed using data collected during the cosmic ray campaign in February 2015 and March 2015. The IBL hit resolution has been improved to 22 (82) $\mu \mathrm{m}$ in local $x$ (local $y$ ). The resolution of the track parameters has been measured by comparing the perigee parameters of two segments of a split cosmic track. During this commissioning phase, a mechanical distortion of the IBL staves was observed. This distortion is caused by the difference in the coefficients of thermal expansion of the IBL components. Using cosmic ray data the magnitude of the distortion has been found to be linearly dependent on the operating temperature with a slope of $\sim 10 \mu \mathrm{m} / \mathrm{K}$. The expected bias on the transverse impact parameter $d_{0}$ during collisions data taking at $\sqrt{s}=13 \mathrm{TeV}$, is expected to be much smaller than its resolution, in the case of temperature fluctuations of $0.2 \mathrm{~K}$.

\footnotetext{
${ }^{2}$ This value represents the expected temperature fluctuation during collisions data taking. The size of this temperature fluctuation was evaluated using the temperature monitoring data during cosmic ray data taking.
} 


\section{References}

[1] ATLAS Collaboration, The ATLAS Experiment at the CERN Large Hadron Collider, JINST 3 S08003 (2008).

[2] ATLAS Collaboration, ATLAS Inner Detector: Technical Design Report 1, ATLAS-TDR-4, CERN-LHCC-1997-016, Geneva:CERN (1997) http://cds.cern.ch/record/331063.

[3] ATLAS Collaboration, ATLAS Insertable B-Layer Technical Design Report, ATLAS-TDR-19, CERN-LHCC-2010-013 (2010) https://cds.cern.ch/record/1291633.

[4] ATLAS Collaboration, ATLAS Insertable B-Layer Technical Design Report Addendum, ATLAS-TDR-19, CERN-LHCC-2010-013 (2010) https://cds.cern.ch/record/1451888.

[5] Butti P. on behalf of ATLAS Collaboration, "Advanced alignment of the ATLAS tracking system", Proceedings of International Conference on High Energy Physics, ATL-PHYS-PROC-2014-231 (2014) (to be published) https://cds.cern.ch/record/1957194.

[6] Jimenez Pena J. on behalf of ATLAS Collaboration, "Alignment of the ATLAS Inner Detector Upgraded for the LHC Run II", ATL-SOFT-PROC-2015-018 (2015) https://cds.cern.ch/record/2015227.

[7] ATLAS Collaboration, "Alignment of the ATLAS Inner Detector and its Performance in 2012", ATLAS-CONF-2014-047 (2014) https://cds.cern.ch/record/1741021.

[8] ATLAS Collaboration, ATLAS Inner Detector Alignment Performance with February 2015 Cosmic Ray Data, ATL-PHYS-PUB-2015-009 (2015) https://cds.cern.ch/record/2008724.

[9] ATLAS Collaboration, Study of the mechanical stability of the ATLAS Insertable B-Layer, ATL-INDET-PUB-2015-001 (2015) https://cds.cern.ch/record/2022587. 